\title{
SUPPLEMENTAL WORKSHEET: GABAY SA PAGKATUTO AT PAG-UNAWA NG SELF- LEARNING MODULE SA FILIPINO
}

\author{
Marielle P. Aro \\ Laguna State Polytechnic University
}

Article DOI: https://doi.org/10.36713/epra7738

DOI No: 10.36713/epra7738

ABSTRAK
Ang pag-aaral na ito ay naglalayong mabatid ang antas ng paggamit ng supplemental worksheet bilang gabay sa
pagkatuto at pag-unawa ng self-learning module sa Filipino. Ginamit ng mananaliksik ang deskriptibong
pamamaraan upang makapangalap ng impormasyon na kaugnay sa pag-aaral. Ang mananaliksik ay gumamit ng
talatanungan at pagsusulit na nakapaloob sa supplemental worksheet na nagsilbing instrumento upang malaman ang
datos na may kaugnayan sa antas ng paggamit batay sa nilalaman, organisasyon/pagkakabuo at kaangkupan ng
gawain. Pagganap batay sa pagsusulit. Lebel ng pagganap sa supplemental worksheet. Kaugnayan ng talatanungan
sa pagganap sa supplemental worksheet at kaugnayan ng pagsusulit sa pagganap sa supplemental worksheet. Ang
nakalap na datos ay tinipon at sinuri sa pamamagitan ng istatistikal na pamamaraan tulad ng mean, standard
deviation at spearman rho upang malaman ang kaugnayan ng talatanungan at pagsusulit sa pagganap sa
supplemental worksheet.
Batay sa datos ng makabuluhang kaugnayan ng gabay sa pagkatuto at pag-unawa sa self-learning module sa
Filipino sa pamamagitan ng talatanungan at pagsusulit sa lebel ng pagganap ng mga mag-aaral sa supplemental
worksheet lumabas na ito ay may kaugnayan. Nangangahulugan lamang ito na naging epektibo ang paggamit ng
binuong supplemental worksheet sa pagpapataas ng lebel ng pagganap ng mag-aaral sa pamamagitan ng
talatanungan at epektibong pagsasagot sa pagsusulit na nakalahad sa supplemental worksheet.
Iminumungkahi ng mananaliksik na ang mga binuong supplemental worksheet para sa mga mag-aaral ng
ikapitong antas ay maaaring gamitin bilang pantulong sa pagtuturo at pagkatuto sa asignaturang Filipino hindi
lamang sa distance learning pati na rin sa harapang pagtuturo.
Hinihikayat ang susunod na bubuo ng kaugnay na pananaliksik ang paggamit ng mas maraming
supplemental worksheet gayundin ang paggamit ng mas maraming tagatugon upang maging batayan ng mas malalim
na pag-aaral na makatutulong sa pagpapaunlad ng kagamitang pampagkatuto.
KEYWORDS: self-learning module, supplemental worksheet, pagganap

\section{PANIMULA}

"Wala man sa silid-aralan, kaisipan ay patuloy na yayabong at mapayayaman".

Itinuturing na ikalawang tahanan na bukas para sa lahat. Guro ang nagsisilbing ama o ina sa loob ng silidaralan. Pumapanday ng kaalaman, nagbibigay inspirasyon at gumagabay sa paghubog ng mga mag-aaral upang maging kapaki-pakinabang sa lipunang kinabibilangan. Isa sa pinakamahalagang bahagi ng lipunan ngunit paano kung ang institusyon na ito ay mahinto? Maipagpapatuloy pa ba ang pag-aaral? 


\section{SJIF Impact Factor 2021: 8.013| ISI I.F.Value:1.241| Journal DOI: 10.36713/epra2016 ISSN: 2455-7838(Online) EPRA International Journal of Research and Development (IJRD)

Ang pagtuturo at pagkatuto ay patuloy na nagbabago habang lumilipas ang panahon batay sa pangangailangan ng mag-aaral na aangkop sa kanilang pag-unawa. Gumagamit ng iba't ibang estratehiya at pamamaraan upang maging mabisa, mas mailapit at maipaunawa ang mga aralin pasalita man ito o pasulat. Ngunit isang malaking hakbang ng pagbabago ang naganap hindi lamang sa sektor ng edukasyon pati na rin sa iba pang larangan dahil sa pandemya ng COVID-19.

Nagpatupad ng iba't ibang kaparaanan ang kagawaran ng edukasyon upang maipagpatuloy ang panuruang taon. Ito ang distance learning kung saan nagaganap ang pagtuturo at pagkatuto kahit hindi pisikal na magkasama ang guro at mag-aaral. Isa sa mga uri nito ay modular modality kung saan gagamit ang mag-aaral ng SLM o SelfLearning Module na naglalaman ng mga aralin sa bawat asignatura sa bawat markahan.

Lahat ay bago sa sistemang ito kung kaya't nagkakaroon ng pagkaantala sa pagdating sa mga paaralan ng module. Upang makatugon sa pangangailangang ito ang guro ay gumagawa ng hakbang sa pamamagitan ng paggawa ng activity worksheet na naglalaman ng mga aralin na dapat matutuhan sa linggong iyon gayundin ang mga gawain o sasagutan ng mag-aaral. Nakabatay sa MELCs o Most Essential Learning Competencies ang mga aralin upang matiyak na ito ay nakaangkla sa itinadhana ng kagawaran. Nakahanay ito sa pambansang pamantayan o balangkas na holistik na Pilipinong mag-aaral na may 21 st siglong kakayahan at ito ay naaangkop sa mga sitwasyon sa totoong buhay.

Ayon sa pananaliksik na isinagawa nina Prasetyo et al. (2018), nagkaroon ng positibong epekto ang paggamit ng worksheet ng kanilang mag-aaral dahil nakapukaw ito ng atensyon, malikhain at makabago na naghatid ng makabuluhang pagkatuto.

Nakalipas na ang dalawang markahan sa taong panuruan ngunit ang mga mag-aaral ay nagkakaroon pa rin ng mababang puntos sa kanilang pagsasagot. Ang mag-aaral na nakararanas ng hirap sa pag-unawa ng aralin ay nangangailangan ng pokus at atensyon mula sa guro upang ito ay matugunan at magabayan kung kaya't kailangang gumamit ng mga pantulong sa pagtuturo. Ito ay mahalaga upang matamo ang layunin na nais ipabatid ng bawat aralin. Ayon sa pag-aaral nina Ulas, et al. (2012) malaki ang epekto ng paggamit ng worksheet sa klase dahil matagumpay itong nakapagpataas ng interes at pagkatuto sa paksa o aralin na kanilang tinalakay.

Sa pag-aaral na ito, nais malaman ng mananaliksik ang antas ng pagkatuto at pag-unawa sa self-learning module sa filipino gamit ang supplemental worksheet. Napili itong paksa ng mananaliksik sapagkat napapanahon at ito ang realidad ng sitwasyon sa mga pampublikong paaralan na humahanap ng iba't ibang estratehiya upang mabilis na maipaunawa ang mga aralin at maihatid ang edukasyon sa gitna ng krisis sa panahon ng pandemya.

\section{Layunin}

Ang pag-aaral na ito ay nagnanais na alamin ang mga sumusunod na pahayag:

1. Ano ang antas ng paggamit ng mga tagatugon sa binuong supplemental worksheet gabay sa pagkatuto at pag unawa sa self-learning module sa Filipino sa pamamagitan ng talatanungan batay sa:

1.1 nilalaman;

1.2 organisasyon/pagkakabuo; at

1.3 kangkupan ng gawain?

2. Ano ang pagganap ng mga mag-aaral batay sa pagsusulit?

3. Ano ang lebel ng pagganap ng mga mag-aaral sa supplemental worksheet?

4. Mayroon bang makabuluhang kaugnayan ang gabay sa pagkatuto at pag unawa sa self-learning module sa Filipino sa pamamagitan ng talatanungan sa lebel ng pagganap ng mga mag-aaral sa supplemental worksheet?

5. Mayroon bang makabuluhang kaugnayan ang pagganap ng mga mag-aaral batay sa pagsusulit sa supplemental worksheet?

\section{METODOLOHIYA \\ Disenyo ng Pananaliksik}

Ang isinagawang pag-aaral ay gumamit ng deskriptibong metodolohiya ng pananaliksik. Ang Palarawan/Deskriptibong Metodolohiya (Descriptive Method) ay idinesenyo para sa mananaliksik tungkol sa isang kalagayan sa kasalukuyan. Upang malaman ang antas ng pagkatuto at pag-unawa sa self-learning module sa filipino gamit ang supplemental worksheet.

Ang deskriptib na pananaliksik ayon kay Badayos (2012) ay isang disenyong pangangalap ng impormasyon hinggil sa mga salik na kaugnay ng paksa ng pananaliksik. 


\section{SJIF Impact Factor 2021: 8.013| ISI I.F.Value:1.241| Journal DOI: 10.36713/epra2016 $\quad$ ISSN: 2455-7838(Online) EPRA International Journal of Research and Development (IJRD)}

\section{Tagatugon sa Pananaliksik}

Ang naging tagatugon sa pananaliksik na ito ay mga piling mag-aaral sa Dayap National High School-Main na nasa ikapitong antas. Ang mga tagatugon ay binubuo ng limampung (50) mag-aaral na binubuo ng dalawampu't limang (25) mag-aaral at dalawampu't limang babae (25) na sasagot sa talatanungan. Ito ay nakabatay sa mga magaaral sa seksyon na hawak ng mananaliksik.

\section{Sampling Teknik}

Ang mananaliksik ay gumamit ng "non-probability sampling technique" na gumagamit ng "nonrandomized" na paraan upang makuha ang magiging tagatugon sa pananaliksik. Isa na rito ang "purposive sampling" kung saan ang tagatugon ay pipiliin batay sa sariling palagay ng mananaliksik. Hindi gaanong gugugol sa pinansyal na aspeto sa sampling teknik na ito. (Showkat, et al. 2017)

\section{Paraan ng Pananaliksik}

Ang mananaliksik ay nagsimula sa pag-iisip at pagtukoy ng mga suliranin na kinahaharap ng mag-aaral, guro, magulang, at paaralan partikular sa kanyang pinaglilingkurang paaralan. Nabatid niya na may suliranin sa aspeto ng kagamitang pampagkatuto na may malaking epekto para sa mag-aaral. Ang pagkaantala ng pagdating ng module na ginagamit ng mga mag-aaral sa "modular distance learning" ay malaking suliranin dahil ito ay napakahalaga sa pagkamit ng pagkatuto sa bawat aralin. Nagkaroon ng ideya ang mananaliksik na gawin ang pagaaral tungkol sa pagtugon sa kakulangan sa module at ito ay ang pagbuo ng "supplemental worksheet".

Ang mananaliksik ay nagsuri ng mga kagamitang pampagtuturo at "self-learning module" na ginamit sa pagbuo ng supplemental worksheet. Pinagbatayan rin ng mananaliksik ang Melc o "Most Essential Learning Competencies" upang matiyak na ang lalamanin ng supplemental worksheet ay naaayon sa balangkas na itinadhana ng kagawaran. Matapos na makapangalap ng mga gagamitin sa pagbuo ng supplemental worksheet ay ang pagpaplano at pagbuo nito. Gumawa ng liham na humingi ng permiso sa pagsasagawa ng pananaliksik sa "Schools Division Superintendent" ng dibisyon gayundin sa punong-guro ng paaralan. Matapos itong mapirmahan at maaprubahan ay sumunod ang pagbuo ng talatanungan na nahahati sa tatlong bahagi: a. Nilalaman $b$. Organisasyon/Pagkakabuo at c. Pagiging angkop ng mga gawain gayundin ang pagbalangkas at pagbuo ng supplemental worksheet na ibibigay pagkatapos ng pagsasagot. Sumunod rito ang pagpapabalida ng binuong supplemental worksheet sa mga guro sa Filipino ng paaralan kung saan nagtuturo ang mananaliksik. Ang mananaliksik ay makakapangalap ng datos batay sa puntos na makukuha ng tagatugon sa supplemental worksheet. Sa huling bahagi ay paghahambing ng isinagawang pagsusulit at balidasyon ng resulta.

\section{Istatistikal na Pagsusuri ng Datos}

Sa pagtukoy sa resulta ng antas ng paggamit ng mga tagatugon batay sa binuong supplemental worksheet gabay sa pagkatuto at pag-unawa sa "self-learning module" sa Filipino sa pamamagitan ng talatanungan batay sa nilalaman, organisasyon at kaangkupan ng gawain, gumamit ng mean at standard deviation ang mananaliksik

Gumamit ng weighted mean at standard deviation sa katanungan para sa pagtukoy ng pagganap ng magaaral batay sa pagsusulit. Gayundin ang ginamit na paraan sa pagkuha ng lebel ng pagganap ng mag-aaral sa supplemental worksheet.

Gumamit ng Spearman rho sa katanungan sa makabuluhang kaugnayan ng talatanungan sa pagganap ng mag-aaral sa "supplemental worksheet" sa gabay sa pagkatuto at pag-unawa ng self-learning module sa Filipino. Gayundin ang ginamit na paraan upang makuha ang datos para sa katanungang makabuluhang kaugnayan ng pagsusulit sa pagganap ng mag-aaral sa supplemental worksheet sa gabay sa pagkatuto at pag-unawa ng selflearning module sa Filipino.

\section{Kinalabasan}

Batay sa mga datos na nakalap mula sa mga piling mag-aaral ng ikapitong (7) baitang ng Dayap National High School-Main

Lumabas sa resulta ng antas ng paggamit ng mga tagatugon sa binuong supplemental worksheet gabay sa pagkatuto at pag-unawa sa self-learning module sa Filipino sa pamamagitan ng talatanungan batay sa nilalaman ay may puna na lubos na sumasang-ayon at may literal na paliwanag na lubhang mataas na antas. 


\section{SJIF Impact Factor 2021: 8.013| ISI I.F.Value:1.241| Journal DOI: 10.36713/epra2016 ISSN: 2455-7838(Online) EPRA International Journal of Research and Development (IJRD)

Batay sa resulta, ang antas ng paggamit ng mga tagatugon sa binuong supplemental worksheet gabay sa pagkatuto at pag-unawa sa self-learning module sa Filipino sa pamamagitan ng talatanungan batay sa organisasyon/pagkakabuo ay may puna na lubos na sumasang-ayon at literal na paliwanag na lubhang mataas.

Ayon sa resulta, ang antas ng paggamit ng mga tagatugon sa binuong supplemental worksheet gabay sa pagkatuto at pag-unawa sa self-learning module sa Filipino sa pamamagitan ng talatanungan batay sa kaangkupan ng gawain ay may puna na lubos na sumasang-ayon at literal na paliwanag na lubhang mataas.

Sang-ayon sa resulta, ang pagganap ng mga mag-aaral batay sa pagsusulit sa tatlong (3) supplemental worksheet ay may puna na mapaglarawang katumbas na may sapat na husay .

Lumabas sa resulta, ang lebel ng pagganap ng mga mag-aaral batay sa supplemental worksheet ay may puna na mapaglarawang katumbas na may sapat na husay.

Nang kinuha ang mean, standard deviation, Spearman rho at puna nakitang may makabuluhang kaugnayan ang supplemental worksheet bilang gabay sa pagkatuto at pag-unawa ng self-learning module sa Filipino.

\section{Rekomendasyon}

Matapos ang pag-aaral at pagsusuri ng mga natuklasan, iminumungkahi ng mananaliksik ang sumusunod na rekomendasyon.

1. Ang mga binuong supplemental worksheet para sa mga mag-aaral ng ikapitong (7) antas ay maaaring gamitin bilang pantulong sa pagtuturo at pagkatuto sa asignaturang Filipino hindi lamang sa distance learning pati na rin sa harapang pagtuturo.

2. Dahil sa naging positibong pagtanggap ng mag-aaral sa pagganap batay sa pagsusulit iminumungkahi na ito ay ipagpatuloy at paunlarin pa sa mga susunod na pag-aaral upang makamit ang mas mataas na pagganap ng mag-aaral sa pagsusulit.

3. Ang mga guro hindi lamang sa asignaturang Filipino ay hinihikayat na tumuklas pa ng iba pang dulog at estratehiya gayundin ang iba pang balangkas na makapagpapaunlad pa ng pagbuo ng mga kagamitang pampagkatuto. Malaki ang maitutulong nito sa pagbabahagi ng kaalaman, pagtugon sa pangangailangan, interes at hikayat sa pagkatuto ng mag-aaral.

4. Hinihikayat ang susunod na bubuo ng kaugnay na pananaliksik ang paggamit ng mas maraming supplemental worksheet gayundin ang paggamit ng mas maraming tagatugon upang maging batayan ng mas malalim na pag-aaral na makatutulong sa pagpapaunlad ng kagamitang pampagkatuto.

5. Hinihikayat ang mga sumusunod na mananaliksik na huwag hihinto sa pagtuklas ng bagong kaalaman o estratehiya pagdating sa usaping pampagtuturo. Ipagpatuloy lamang ang pag-aaral na ito upang dumami pa ang matuklasan na kaugnayan ng mga datos na siyang magsisilbing tulong sa maaari pang ikaunlad ng pananaliksik na ito.

\section{Konklusyon}

Sa pamamagitan ng mga inilahad na pagbubuo, nabuo ang sumusunod na konklusyon.

1. Batay sa datos ng makabuluhang kaugnayan ng gabay sa pagkatuto at pag-unawa sa self-learning module sa Filipino sa pamamagitan ng talatanungan at pagsusulit sa lebel ng pagganap ng mga mag-aaral sa supplemental worksheet lumabas na ito ay may kaugnayan. Nangangahulugan lamang ito na naging epektibo ang paggamit ng binuong supplemental worksheet sa pagpapataas ng lebel ng pagganap ng mag-aaral sa pamamagitan ng talatanungan at epektibong pagsasagot sa pagsusulit na nakalahad sa supplemental worksheet.

2. Sang-ayon sa resulta ng makabuluhang kaugnayan ng pagganap ng mga mag-aaral batay sa pagsusulit sa lebel ng pagganap ng mga mag-aaral sa supplemental worksheet lumabas na ito ay may kaugnayan batay sa antas ng kabuluhan. Nangangahulugan lamang ito na naging positibo ang paggtanggap ng mag-aaral na nagresulta sa epektibong pagsasagot sa pagsusulit na nakalahad sa supplemental worksheet.

\section{Pigura, talahanayan at talasanggunian}

Talahanayan 1. Antas ng paggamit ng mga tagatugon sa binuong supplemental worksheet gabay sa pagkatuto at pagunawa ng self-learning module sa Filipino sa pamamagitan ng talatanungan batay sa nilalaman 
SJIF Impact Factor 2021: 8.013| ISI I.F.Value:1.241| Journal DOI: 10.36713/epra2016

ISSN: 2455-7838(Online)

EPRA International Journal of Research and Development (IJRD)

Volume: 6 | Issue: 7 | July 2021

- Peer Reviewed Journal

\begin{tabular}{|c|c|c|c|}
\hline Mga Pahayag & Mean & SD & Puna \\
\hline Malinaw na nailahad ang mga detalye ng paksa. & 4.70 & 0.46 & Lubos na sumasang-ayon \\
\hline Madaling maunawaan ang nilalaman. & 4.54 & 0.58 & Lubos na sumasang-ayon \\
\hline Nakapupukaw ng interes ang paggamit ng larawan. & 4.60 & 0.53 & Lubos na sumasang-ayon \\
\hline $\begin{array}{l}\text { Nakapagpalinaw ng impormasyon ang paggamit ng graphic } \\
\text { organizer. }\end{array}$ & 4.50 & 0.61 & Lubos na sumasang-ayon \\
\hline Nakasusunod sa MELCs ang aralin. & 4.30 & 0.65 & Lubos na sumasang-ayon \\
\hline Nalilinang ang kritikal na pag-iisip. & 4.28 & 0.61 & Lubos na sumasang-ayon \\
\hline Nakapagbibigay ng malinaw na konsepto ng paksa. & 4.52 & 0.50 & Lubos na sumasang-ayon \\
\hline $\begin{array}{l}\text { Bukas ang isipan sa pagbabagong nagaganap sa proseso } \\
\text { ng pagkatuto sa kasalukuyang panahon. }\end{array}$ & 4.46 & 0.61 & Lubos na sumasang-ayon \\
\hline $\begin{array}{l}\text { Madaling nagagamit ang supplemental worksheet sa } \\
\text { pagtugon sa pangangailangan ng mag-aaral sa pagbuo ng } \\
\text { pananaliksik }\end{array}$ & 4.38 & 0.67 & Lubos na sumasang-ayon \\
\hline Nakatutulong sa pagpapataas ng antas ng kaalaman. & 4.48 & 0.61 & Lubos na sumasang-ayon \\
\hline \multicolumn{4}{|l|}{$\begin{array}{l}\text { Overall Mean: } 4.48 \\
\text { Standard Deviation: } 0.595 \\
\text { Literal na paliwanag: } \text { Lubhang Mataas }\end{array}$} \\
\hline
\end{tabular}

Sa lubhang mataas na antas ng paggamit at sa pananaw ng mga tagatugon, may malinaw na nailahad ang mga detalye ng paksa ay nakakuha ng $(\mathrm{M}=4.70, \mathrm{SD}=0.46)$ at nakapupukaw ng interes ang paggamit ng larawan ay mayroong $(\mathrm{M}=4.60, \mathrm{SD}=0.53)$. Bagaman napansin na lubhang mataas na antas ng paggamit, ang pahayag na may pinakamababang marka ay nalilinang ang kritikal na pag-iisip ay mayroong $(\mathrm{M}=4.28, \mathrm{SD}=0.61)$.

May kabuuang mean na 4.48, standard deviation na 0.595 at ipinapakita na ang antas ng paggamit ng mga tagatugon sa binuong supplemental worksheet gabay sa pagkatuto at pag-unawa ng self-learning module sa Filipino sa pamamagitan ng talatanungan batay sa nilalaman ay may puna na lubos na sumasang-ayon at literal na paliwanag na lubhang mataas.

Talahanayan 2. Antas ng paggamit ng mga tagatugon sa binuong supplemental worksheet gabay sa pagkatuto at pagunawa ng self- learning module sa Filipino sa pamamagitan ng talatanungan batay sa organisasyon/pagkakabuo

\begin{tabular}{|l|c|c|c|}
\hline Mga Pahayag & Mean & SD & Puna \\
\hline Nailahad ang paksa sa paraang lohikal. & 4.24 & 0.72 & Lubos na sumasang-ayon \\
\hline Maayos na paghahanay ng impormasyon. & 4.52 & 0.65 & Lubos na sumasang-ayon \\
\hline Sapat ang mga impormasyon na inilahad. & 4.50 & 0.68 & Lubos na sumasang-ayon \\
\hline Mabilis maunawan ang mga salitang ginamit. & 4.48 & 0.71 & Lubos na sumasang-ayon \\
\hline Pormal at hindi maligoy. & 4.48 & 0.71 & Lubos na sumasang-ayon \\
\hline Malayang nakasusunod sa instruksyon ng aralin. & 4.46 & 0.73 & Lubos na sumasang-ayon \\
\hline
\end{tabular}




\section{SJIF Impact Factor 2021: 8.013| ISI I.F.Value:1.241| Journal DOI: 10.36713/epra2016 ISSN: 2455-7838(Online)}

EPRA International Journal of Research and Development (IJRD)

\begin{tabular}{|l|c|c|c|}
\hline $\begin{array}{l}\text { Mabilis na naiuugnay ang dati nang kaalaman sa panibagong } \\
\text { impormasyon. }\end{array}$ & 4.32 & 0.68 & Lubos na sumasang-ayon \\
\hline Mabisang nailahad ang pagkakasunod-sunod ng paksa. & 4.44 & 0.64 & Lubos na sumasang-ayon \\
\hline Malinaw ang daloy ng paghahanay ng impormasyon. & 4.48 & 0.65 & Lubos na sumasang-ayon \\
\hline Organisado ang pagkakabuo ng supplemental worksheet. & 4.40 & 0.73 & Lubos na sumasang-ayon \\
\hline $\begin{array}{l}\text { Overall Mean: } 4.43 \\
\text { Standard Deviation: } 0.689 \\
\text { Literal na paliwanag: Lubhang Mataas }\end{array}$ & & \\
\hline
\end{tabular}

Sa lubhang mataas na antas ng paggamit at sa pananaw ng mga tagatugon, may maayos na paghahanay ng impormasyon ay nakakuha ng $(\mathrm{M}=4.52, \mathrm{SD}=0.65)$ at sapat ang mga impormasyon na inilahad ay mayroong $(\mathrm{M}=$ 4.50, SD = 0.68). Bagaman napansin na lubhang mataas na antas ng paggamit, ang pahayag na may pinakamababang marka ay nailahad ang paksa sa paraang lohikal ay mayroong $(\mathrm{M}=4.24, \mathrm{SD}=0.72)$.

May kabuuang mean na 4.43 , standard deviation na 0.689 at ipinapakita na ang antas ng paggamit ng mga tagatugon sa binuong supplemental worksheet gabay sa pagkatuto at pag unawa ng self-learning module sa Filipino sa pamamagitan ng talatanungan batay sa organisasyon/pagkakabuo ay may puna na lubos na sumasang-ayon at literal na paliwanag na lubhang mataas.

Talahanayan 3. Antas ng paggamit ng mga tagatugon sa binuong supplemental worksheet gabay sa pagkatuto at pagunawa ng self-learning module sa Filipino sa pamamagitan ng talatanungan batay sa kaangkupan ng mga gawain

\begin{tabular}{|c|c|c|c|}
\hline Mga Pahayag & Mean & SD & Puna \\
\hline Madaling sagutan ang gawain. & 4.40 & 0.73 & Lubos na sumasang-ayon \\
\hline $\begin{array}{l}\text { Ang mga tanong na nakalagay ay sapat upang maunawaan ang } \\
\text { paksa. }\end{array}$ & 4.52 & 0.58 & Lubos na sumasang-ayon \\
\hline $\begin{array}{l}\text { Nakatulong ang paggamit ng larawan sa pagbibigay ng } \\
\text { impormasyon. }\end{array}$ & 4.56 & 0.50 & Lubos na sumasang-ayon \\
\hline Nakapagpalawak ng kaisipan at interes. & 4.52 & 0.61 & Lubos na sumasang-ayon \\
\hline Naging kasiya-siya ang naging pagkatuto. & 4.54 & 0.71 & Lubos na sumasang-ayon \\
\hline Mabilis na nakatugon at nakabuo ng kasagutan. & 4.30 & 0.74 & Lubos na sumasang-ayon \\
\hline $\begin{array}{l}\text { Nailalapat ang natamong kaalaman sa aralin sa totoong } \\
\text { sitwasyon ng buhay. }\end{array}$ & 4.26 & 0.72 & Lubos na sumasang-ayon \\
\hline Napauunlad ang kakayahan sa malayang pagkatuto. & 4.46 & 0.65 & Lubos na sumasang-ayon \\
\hline $\begin{array}{l}\text { Napauunlad ang kasanayan sa pagsulat gamit ang } \\
\text { supplemental worksheet. }\end{array}$ & 4.44 & 0.64 & Lubos na sumasang-ayon \\
\hline $\begin{array}{l}\text { Nakabubuo ng repleksyon sa aralin matapos gamitin ang } \\
\text { supplemental worksheet. }\end{array}$ & 4.30 & 0.79 & Lubos na sumasang-ayon \\
\hline \multicolumn{4}{|l|}{$\begin{array}{l}\text { Overall Mean: } 4.43 \\
\text { Standard Deviation: } 0.674 \\
\text { Literal na paliwanag: Lubhang Mataas }\end{array}$} \\
\hline
\end{tabular}


Sa lubhang mataas na antas ng paggamit at sa pananaw ng mga tagatugon, nakatulong ang paggamit ng larawan sa pagbibigay ng impormasyon ay nakakuha ng $(\mathrm{M}=4.56, \mathrm{SD}=0.50)$ at naging kasiya-siya ang naging pagkatuto ay mayroong $(\mathrm{M}=4.54, \mathrm{SD}=0.71)$. Bagaman napansin na lubhang mataas na antas ng paggamit, ang pahayag na may pinakamababang marka ay nailalapat ang natamong kaalaman sa aralin sa totoong sitwasyon ng buhay ay mayroong $(\mathrm{M}=4.26, \mathrm{SD}=0.72)$.

May kabuuang mean na 4.43, standard deviation na 0.674 at ipinapakita na ang antas ng paggamit ng mga tagatugon sa binuong supplemental worksheet gabay sa pagkatuto at pag-unawa sa self-learning module sa Filipino sa pamamagitan ng talatanungan batay sa kaangkupan ng mga gawain ay may puna na lubos na sumasang-ayon at literal na paliwanag na lubhang mataas.

Talahanayan 4. Pagganap ng mga mag-aaral batay sa pagsusulit

\begin{tabular}{|l|c|c|c|c|c|c|}
\hline Written & $\begin{array}{c}\text { Pinakamababang } \\
\text { Marka }\end{array}$ & $\begin{array}{c}\text { Pinakamataas na } \\
\text { marka }\end{array}$ & Mean & SD & \% & $\begin{array}{c}\text { Mapaglarawang } \\
\text { Katumbas }\end{array}$ \\
\hline $\begin{array}{l}\text { Worksheet 1 } \\
(11 \text { items })\end{array}$ & 5 & 11 & 9.64 & 1.588 & 87.64 & $\begin{array}{c}\text { Higit na } \\
\text { mahusay }\end{array}$ \\
\hline $\begin{array}{l}\text { Worksheet 2 } \\
(19 \text { items })\end{array}$ & 8 & 19 & 14.40 & 3.136 & 75.79 & Mahusay \\
\hline $\begin{array}{l}\text { Worksheet 3 } \\
(23 \text { items })\end{array}$ & 12 & 23 & 18.02 & 3.100 & 78.35 & Mahusay \\
\hline Overall & $\mathbf{5}$ & $\mathbf{2 3}$ & $\mathbf{1 4 . 0 2}$ & $\mathbf{4 . 3 6 8}$ & $\mathbf{8 0 . 5 9}$ & Mahusay \\
\hline
\end{tabular}

Talahanayan bilang apat ay nagpapakita ng pagganap ng mga mag-aaral batay sa pagsusulit, sa kabuuang limampung tagatugon, ang "Written Work 1" ay nakakuha ng $(\mathrm{M}=9.64, \mathrm{SD}=1.588)$ at may pinakamataas na bahagdan na $87.64 \%$ ng kabuuang tumugon at may mapaglarawang katumbas na Higit na mahusay. At ang "Written Work 3" ay nakakuha ng $(\mathrm{M}=18.02, \mathrm{SD}=3.100)$ at may mayroong bahagdan na $78.35 \%$ ng kabuuang tumugon at may mapaglarawang katumbas na Mahusay. Habang ang "Written Work 2" ay nakakuha ng $(\mathrm{M}=14.40, \mathrm{SD}=3.316)$ at may pinakamataas na bahagdan na $75.79 \%$ ng kabuuang tumugon at may literal na paliwanag na Mahusay.

May kabuuang (Weighted Mean $=14.02, \mathrm{SD}=4.368$ ) at (pinakamababang marka $=5$, pinakamataas na marka $=23$ ) ipinapakita na ang antas ng pagganap ng mga mag-aaral batay sa pagsusulit ay may literal na paliwanag na Mahusay.

Talahanayan 5. Lebel ng pagganap ng mga mag-aaral sa supplemental worksheet

\begin{tabular}{|l|c|c|c|c|c|}
\hline Performance & $\begin{array}{c}\text { Pinakamababang } \\
\text { Marka }\end{array}$ & $\begin{array}{c}\text { Pinakamataas na } \\
\text { marka }\end{array}$ & Mean & $\begin{array}{c}\text { Standard } \\
\text { Deviation }\end{array}$ & $\begin{array}{c}\text { Mapaglarawang } \\
\text { Katumbas }\end{array}$ \\
\hline $\begin{array}{l}\text { Task 1 } \\
(15 \text { items })\end{array}$ & 7 & 15 & 11.54 & 2.270 & Mahusay \\
\hline $\begin{array}{l}\text { Task 2 } \\
(15 \text { items })\end{array}$ & 7 & 15 & 11.84 & 2.351 & Mahusay \\
\hline $\begin{array}{l}\text { Task 3 } \\
(15 \text { items })\end{array}$ & 8 & 15 & 12.00 & 2.466 & Mahusay \\
\hline Overall & 7 & $\mathbf{1 5}$ & $\mathbf{1 1 . 7 9}$ & $\mathbf{2 . 3 5 5}$ & Mahusay \\
\hline
\end{tabular}

Talahanayan bilang lima ay nagpapakita ng lebel ng pagganap ng mga mag-aaral sa Supplemental Worksheet, sa kabuuang limampung tagatugon, ang "Performance Task 3" ay nakakuha pinakamataas na (M=12.00, $\mathrm{SD}=2.466)$ at may literal na paliwanag na Mahusay. At ang "Performance Task 2" ay nakakuha ng $(\mathrm{M}=11.84$, 


\section{SJIF Impact Factor 2021: 8.013| ISI I.F.Value:1.241| Journal DOI: 10.36713/epra2016 ISSN: 2455-7838(Online) EPRA International Journal of Research and Development (IJRD)

$\mathrm{SD}=2.351$ ) at may mapaglarawang katumbas na Mahusay. Habang ang "Performance Task 1" ay nakakuha pinakamababa na $(\mathrm{M}=11.54, \mathrm{SD}=2.270)$ at may literal na paliwanag na Mahusay.

May kabuuang (Weighted Mean $=11.79, \mathrm{SD}=2.355$ ) at (pinakamababang marka $=7$, pinakamataas na marka $=15$ ) ipinapakita na ang lebel ng pagganap ng mga mag-aaral sa Supplemental Worksheet, ay may literal na paliwanag na Mahusay.

Talahanayan 6. Makabuluhang kaugnayan ng gabay sa pagkatuto at pag- unawa sa self-learning module sa Filipino sa pamamagitan ng talatanungan sa lebel ng pagganap ng mga mag-aaral sa Supplemental Worksheet

\begin{tabular}{|c|c|c|c|}
\hline $\begin{array}{c}\text { Gabay sa pagkatuto at pag unawa sa } \\
\text { self-learning module sa Filipino }\end{array}$ & Spearman rho & Katibayan ng Ugnayan & Analisis \\
\hline $\begin{array}{l}\text { Lebel ng pagganap ng mga mag-aaral } \\
\text { sa Supplemental Worksheet }\end{array}$ & 0.2347 & Mababang pagkakaugnay & makabuluhan \\
\hline
\end{tabular}

Talahanayan bilang anim ay nagpapakita ng makabuluhang kaugnayan ng gabay sa pagkatuto at pag-unawa sa self-learning module sa Filipino sa pamamagitan ng talatanungan sa lebel ng pagganap ng mga mag-aaral sa Supplemental Worksheet. Ang datos ay ginamitan ng istatistikong pamamaraan na nagresulta ng tinuos na halaga para sa Spearman rho. Ipinapakita dito na ang tinuos na halaga para sa Lebel ng pagganap ng mga mag-aaral sa Supplemental Worksheet, may Spearman rho na 0.2347 ay may mababang pagkakaugnay at mayroong suportang tinuos na Spearman rho at ang pagsusuri ay makabuluhan.

Talahanayan 7. Makabuluhang kaugnayan ng pagganap ng mga mag- aaral batay sa pagsusulit sa Supplemental Worksheet

\begin{tabular}{|l|l|l|c|}
\hline $\begin{array}{c}\text { Performans ng mga mag-aaral batay sa } \\
\text { pagsusulit ayon sa rubrik }\end{array}$ & Spearman rho & Katibayan ng Ugnayan & Analisis \\
\hline $\begin{array}{l}\text { Lebel ng performans ng mga mag-aaral } \\
\text { sa Supplemental Worksheet }\end{array}$ & 0.8987 & Mataas na pagkakaugnay & makabuluhan \\
\hline
\end{tabular}

Talahanayan bilang pito ay nagpapakita ng makabuluhang kaugnayan ng pagganap ng mga mag-aaral batay sa pagsusulit. Ang datos ay ginamitan ng istatistikong pamamaraan na nagresulta ng tinuos na halaga para sa Spearman rho. Ipinapakita dito na ang tinuos na halaga para sa Lebel ng pagganap ng mga mag-aaral sa Supplemental Worksheet, may Spearman rho na 0.8987 ay may mababang pagkakaugnay at mayroong suportang tinuos na Spearman rho at ang pagsusuri ay makabuluhan.

\section{TALASANGGUNIAN}

1. Badayos, P. (2012), "Metodolohiya sa Pagtuturo ng Wika Mga Teorya, Simulain at Estratehiya", Grandhouse Publishing Inc.

2. Prasetyo Z. Mutya N. (2018), "The Effectiveness of Students' Worksheet Basedon MultipleRepresentations to Increase Creative Thinking Skills".Retrieved from http://journal.uad.ac.id/index.php/EduLearn/article/view/8487/pdf_287

3. Showkat, N. Pareveen H. (2017), "Non Probabilty and Probability

4. Sampling".Retrieved from https://www.researchgate.net/publication/319066480

5. Ulas H. (2012), "The effect of worksheets based upon 5e learning cycle model on student success in teaching of adjectives as grammatical components". Retrieved from https://www.researchgate.net/publication/257715100 